\title{
Índices de Simetría Condilar y Mandibular a Través de Radiografías Panorámicas Digitales en una Muestra de Pacientes Chilenos
}

\author{
Condylar and Mandibular Symmetry Indexes Through Digital \\ Panoramic Radiographs in a Sample of Chileans Patients
}

\author{
Ramón Fuentes ${ }^{1,2}$; Manuel Arellano-Villalón ${ }^{3}$; Natalia Soto-Faúndez ${ }^{3}$; \\ Fernando J. Dias ${ }^{1,2}$; Pablo Navarro ${ }^{1,2} \&$ Alain Arias ${ }^{1,2,4}$
}

FUENTES, R.; ARELlanO-VILlalón, M.; SOTO-FAÚNDEZ, N.; DIAS, F. J.; NAVARRO, P. \& ARIAS, A. Índices de simetría condilar y mandibular a través de radiografías panorámicas digitales en una muestra de pacientes chilenos. Int. J. Morphol., 36(3):854$858,2018$.

RESUMEN: La simetría mandibular puede alterarse por diversos motivos. Variados estudios buscan definir parámetros de "normalidad" y para ello se han descrito técnicas que utilizan la radiografía panorámica (RPN). El objetivo de este estudio fue determinar índices de simetría mandibular/condilar en una muestra de pacientes chilenos. Se realizó un estudio descriptivo de corte trasversal en RPN digitales obtenidas de la Clínica Odontológica de la Universidad de La Frontera. Se aplicaron criterios de inclusión: pacientes mayores de 18 años, dentición permanente completa hasta el primer molar; y de exclusión: signos radiográficos de alteraciones óseas y/ o cirugía mandibular, cóndilos no visibles, ortodoncia. Se utilizó el software ImageJ para dibujar rectas y medir la altura de la rama y cóndilo mandibular del lado derecho e izquierdo de acuerdo a la técnica propuesta por Kjellberg et al. (1994). Con estas medidas se calculó el índice de simetría entre altura condilar y rama (S1); y entre altura condilar y mandibular (S2). 235 RPN digitales fueron analizadas (95 hombres; 140 mujeres) con edad promedio de 24,98 $\pm 7,32$ años. El promedio de S1 fue de 93,74\% ( $\pm 3,85)$ en hombres y $93,41 \%( \pm 5,77)$ en mujeres, mientras que para S2 fue de $90,50 \%( \pm 6,51)$ en hombres y $90,27 \%( \pm 7,80)$ en mujeres, sin diferencias significativas para ninguno de ambos índices $(\mathrm{p}=0,347$ y $\mathrm{p}=0,233$, respectivamente). En cuanto a la edad, tampoco se encontraron diferencias significativas en el promedio de los índices S1 y S2 para cada rango etario ( $\mathrm{p}=0,594$ y p=0,669, respectivamente). Mediante este estudio se concluye que hay un alto porcentaje de pacientes con simetría mandibular/condilar en ambos sexos y en diferentes rangos etarios, esto sin considerar el estado de salud o presencia de alteraciones morfológicas y/o funcionales.

PALABRAS CLAVE: Simetría Mandibular; Simetría condilar; Radiografía panorámica digital; Prevalencia.

\section{INTRODUCCIÓN}

Las asimetrías condilares han sido reportadas en diversos estudios asociadas a diferentes alteraciones o estados de anormalidad (Arenas et al., 2012; Iturriaga et al., 2012; Abad-Santamaría et al., 2014; Kasimoglu et al., 2015; Alfaro et al., 2016) como por ejemplo, en limitaciones y desviaciones en la apertura y dolor estático (Bezuur et al., 1988), y en casos de pacientes con mordida cruzada unilateral, mostrando un gran porcentaje de asimetría de cóndilo y rama (Arenas et al.) y estableciéndose como posible factor de riesgo para el desarrollo de trastornos témporomandibulares (Iturriaga et al.).

Habets et al. (1988) investigaron la relación entre las asimetrías condilares y los trastornos témporo- mandibulares, introduciendo un método para determinar la asimetría entre los cóndilos y las ramas mandibulares el cual compara la altura vertical del cóndilo y rama mandibular de los lados izquierdo y derecho en radiografías panorámicas (RPN). Este método, describe que resultados mayores a $3 \%$ indicarían asimetría vertical (Habets et al.). Con respecto a este porcentaje, se encontró que independiente del subdiagnóstico, el $74 \%$ de estos pacientes con trastornos cráneomandibulares tenían una asimetría condilar vertical mayor al $3 \%$, a pesar de que pacientes sin limitaciones de apertura y sin desviaciones demostraran más asimetría que pacientes con limitación y con desviaciones (Benzuur et al.). Kjellberg et al. (1994) desarrollaron un método que resulta independiente del factor de magnificación de la radiografía

\footnotetext{
${ }^{1}$ Departamento de Odontología Integral Adultos, Facultad de Odontología, Universidad de La Frontera, Temuco, Chile.

${ }^{2}$ Centro de Investigación en Ciencias Odontológicas (CICO), Facultad de Odontología, Universidad de La Frontera, Temuco, Chile.

${ }^{3}$ Escuela de Odontología, Facultad de Odontología, Universidad de La Frontera, Temuco, Chile.

${ }^{4}$ Universidad Adventista de Chile, Chillán, Chile.
} 
con el cual determina la altura condilar en RPN y puede ser aplicado en pacientes con o sin lesiones condilares. Estudios han demostrado que esta técnica tiende a la simetría y sus resultados tienen una disparidad más estrecha, disminuyendo la posibilidad de falsos positivos (Fuentes et al., 2011) y sugiriendo su uso en futuros estudios.

Se ha observado en adultos con maloclusiones que permanecen sin tratamiento que hay una remodelación esquelética de la ATM y reposicionamiento adaptativo de la fosa articular en el hueso temporal, por lo que estos eventos serían el motivo por el cual la asimetría mandibular exhibida en la radiografía no se puede asociar a la patología (AbadSantamaría et al.). También se ha observado diferencia de altura entre cóndilos izquierdo y derecho en pacientes con trastornos témporomandibulares, como también en pacientes sin dichos trastornos (Luz et al., 2002). Es por esto, que, a pesar de los numerosos estudios mencionados, muchos sugieren profundizar en el tema con estudios que contengan muestras más numerosas (Ramirez-Yañez et al., 2010; AbadSantamaría et al.; Alfaro et al.) en donde se establezcan parámetros de normalidad de las asimetrías o simetrías utilizando las técnicas ya descritas. Por todo lo anterior, el objetivo de este estudio fue determinar índices de simetría mandibular/condilar en una muestra de pacientes atendidos en la Clínica Odontológica Docente Asistencial (CODA) de la Universidad de La Frontera (Temuco, Chile), utilizando la técnica descrita por Kjellberg en RPN digitales.

\section{MATERIAL Y MÉTODO}

Se realizó un estudio descriptivo de corte trasversal en el que se analizaron 1382 RPN digitales (proporción 1:1) tomadas entre enero del año 2014 y diciembre del año 2015 y que se encontraban disponibles en la CODA de la Facultad de Odontología de la Universidad de La Frontera, Temuco, Chile. Las radiografías se tomaron usando el protocolo estándar con el ortopantomografo PAX-400C (VATECH, Korea, 2010). Este estudio cuenta con la aprobación del Comité Ético Científico (CEC) de la Universidad de La Frontera (Folio n 015/2014).

Se aplicaron los siguientes criterios de inclusión: radiografías de pacientes hombres y mujeres, mayores de 18 años, con dentición permanente completa hasta el primer molar. Del mismo modo se aplicaron los siguientes criterios de exclusión: radiografías con distorsión o alteración de contraste, pacientes con signos o evidencias de alteraciones óseas, signos o evidencias de cirugía ortognática mandibular, cóndilos mandibulares no visibles, pacientes con tratamiento de ortodoncia y presencia de dientes supernumerarios $u$ otra alteración del desarrollo.

Las radiografías seleccionadas fueron analizadas utilizando la técnica de Kjellberg para determinar el porcentaje de simetría condilar y mandibular. Las mediciones fueron realizadas por dos examinadores previamente capacitados con un especialista en radiología oral y maxilofacial para la identificación de puntos anatómicos específicos en la radiografía. Adicionalmente, se realizó una calibración interoperador mediante el cálculo del coeficiente de correlación intraclase (CCI) para las diferentes medidas condilares y mandibulares que considera el método de Kjellberg. Para las mediciones se utilizó el software ImageJ (v. 1.49) que permite trazar rectas y calcular medidas en la radiografía previamente ajustada en la escala de medición. Para determinar las simetrías, en primer lugar se trazó una línea recta tangente que pasara por el punto más lateral del cóndilo y por el punto más lateral de la rama (recta RL). Luego se trazaron dos líneas rectas paralelas y horizontales que fueran tangentes al punto más superior del cóndilo (recta $\mathrm{CO}$ ) y al punto más inferior de la escotadura mandibular (recta $\mathrm{MN}$ ) respectivamente, proyectando ambas hasta interceptar a RL. Adicionalmente, se trazó una línea recta tangente a los dos puntos inferiores más prominentes del cuerpo de la mandíbula (recta ML), proyectándola hasta interceptar con RL. Finalmente, se trazó una línea recta paralela a la horizontal (recta GO) que pasara por el punto de intersección de RL y ML (Fig. 1).

Utilizando las rectas trazadas se determinaron las siguientes medidas: longitud de RL entre $\mathrm{CO}$ y $\mathrm{MN}$ (altura condilar o $\mathrm{CH}$ ), longitud de RL entre MN y GO (altura mandibular o $\mathrm{MH}$ ) y longitud de RL entre CO y GO (altura de la rama o RH) (Fig. 2). Para determinar el porcentaje de simetría condilar y mandibular de la radiografía analizada, se aplicó la fórmula descrita por Kjellberg et al. (Fig. 3) y así se obtuvieron los índices de simetría S1 y S2. Para S1 en el numerador se agrega el valor menor de la división entre $\mathrm{CH}$ y $\mathrm{RH}$ de un lado de la mandíbula y en el divisor, el mayor; esta división se multiplica por 100. Para S2 en el numerador se agrega el valor menor de la división entre $\mathrm{CH}$ y $\mathrm{MH}$ de un mismo lado de la mandíbula y en el divisor, el mayor; esto se multiplica por 100. Para determinar si existe simetría, S1 debe ser mayor a $93 \%$ y S2 mayor a $90 \%$.

Análisis estadístico. El porcentaje de simetría determinado (índices S1 y S2) fue relacionado con el sexo y edad de los pacientes. Para el análisis estadístico se utilizó el software SPSS v20.0 (IBM, SPSS, Chicago, Il, USA). Para la presentación de datos cuantitativos se utilizó la media \pm desviación estándar y se aplicó T- test y ANOVA de un factor para el contraste de variables. Se utilizó un valor $\mathrm{p}<0,05$ como umbral de significancia. 


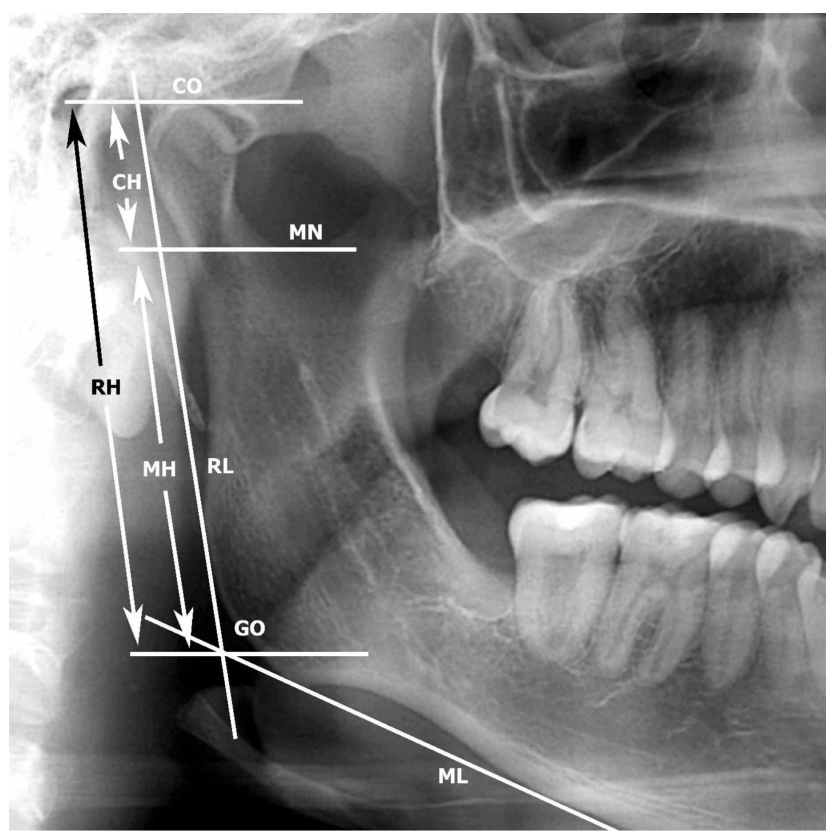

Fig. 1. Líneas trazadas para el método de Kjellberg. RL: línea recta tangente que pasara por el punto más lateral del cóndilo y por el punto más lateral de la rama. CO: línea recta horizontal tangente al punto más superior del cóndilo. MN: línea recta horizontal tangente al punto más inferior de la escotadura mandibular. ML: línea recta tangente a los dos puntos inferiores más prominentes del cuerpo de la mandíbula. GO: línea recta paralela a la horizontal que pase por el punto de intersección de RL y ML. CH: altura condilar. MH: altura mandibular. RH: altura de la rama.

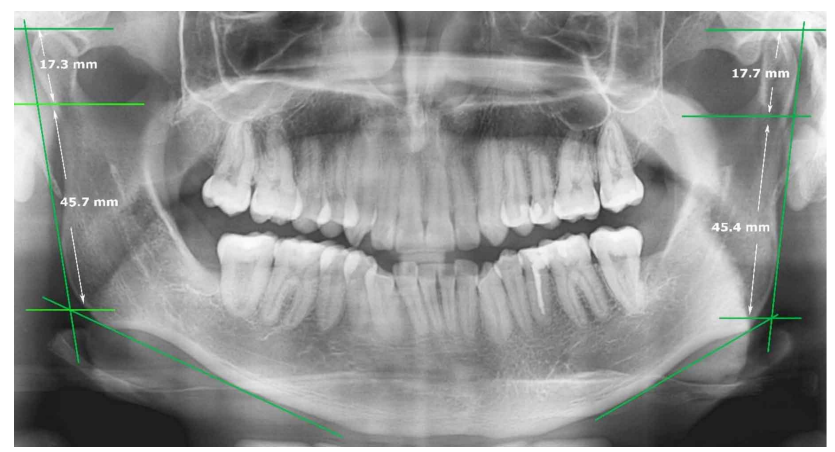

Fig. 2. Ejemplo de medición de altura condilar $(\mathrm{CH})$ y altura mandibular (MH) en una RPN digital según el método de Kjellberg.

\section{RESULTADOS}

En el análisis de la concordancia interoperador, el CCI fue de 95,6\% (muy buena concordancia). Una vez

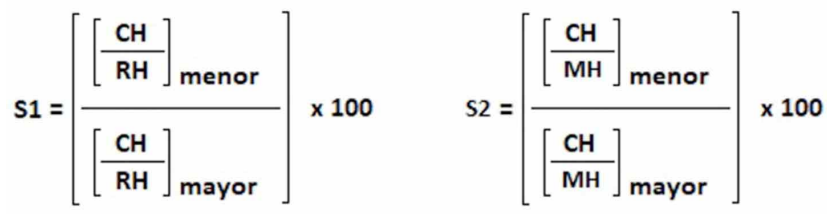

Fig. 3. Fórmula descrita por Kjellberg para calcular porcentajes de simetrías condilares y mandibulares. Para S1 en el numerador se agrega el valor menor de la división entre $\mathrm{CH}$ y $\mathrm{RH}$ de un lado de la mandíbula y en el divisor, el mayor; esta división se multiplica por 100. Para S2 en el numerador se agrega el valor menor de la división entre $\mathrm{CH}$ y $\mathrm{MH}$ de un mismo lado de la mandíbula y en el divisor, el mayor; esto se multiplica por 100. Para determinar si existe simetría, $\mathrm{S} 1$ debe ser mayor a 93 $\%$ y S2 mayor a $90 \%$.

aplicados los criterios de inclusión y exclusión, en total se analizaron 235 RPN digitales, de las cuales 95 radiografías pertenecían a hombres $(40,4 \%)$ y 140 a mujeres $(59,6$

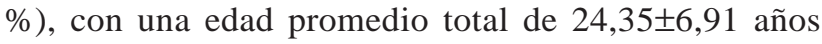

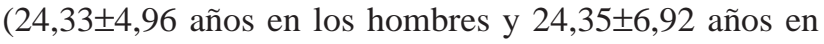
las mujeres). En el total de la muestra el grupo etario predominante fue el de 20 a 29 años, representando un 63,8 $\%(n=150)$.

En total, el promedio de $\mathrm{S} 1$ fue de $93,3 \%( \pm 5,08)$ y el de $\mathrm{S} 2$ fue de $90,7 \%( \pm 6,96)$. En la Tabla I se presentan los promedios de S1 y S2 agrupados según el sexo de los pacientes. En el caso de los hombres $(\mathrm{n}=95)$, el promedio de $\mathrm{S} 1$ fue de $93,7 \%( \pm 3,85)$ y el de $\mathrm{S} 2$ fue $91,3 \%( \pm 5,16)$. En el caso de las mujeres $(\mathrm{n}=140)$, el promedio de $\mathrm{S} 1$ fue de $93,1 \%( \pm 5,77)$ y el de $\mathrm{S} 2$ fue de $90,3 \%( \pm 7,95)$. No se encontraron diferencias significativas en los valores de S1 y S2 entre hombres y mujeres $(\mathrm{p}=0,347$ y $\mathrm{p}=0,233$, respectivamente).

En cuanto a la edad, en la Tabla II se observa que el valor más bajo de $\mathrm{S} 1$ se encontró en el grupo de 50-59 años, siendo $91,43 \%( \pm 3,8)$ y el más alto se encontró en el grupo de 40 - 49 años, siendo 95,57\% $( \pm 3,79)$. En el caso de $\mathrm{S} 2$, valor promedio más bajo fue $88,65 \%( \pm 5,19)$ en el grupo de $50-59$ años y el más alto fue $93,58 \%( \pm 5,4)$ en el grupo de 40-49 años. Al comparar ambos índices entre los diferentes rangos etarios establecidos, no se encontraron diferencias significativas tanto para $\mathrm{S} 1(\mathrm{p}=0,594)$ como para $\mathrm{S} 2(\mathrm{p}=0,669)$.

Tabla I. Promedio y desviación estándar de los índices S1 y S2 agrupados por sexo.

\begin{tabular}{lllllll}
\hline Sexo & $\mathrm{n}$ & $\%$ & $\mathrm{~S} 1(\%)$ & Desv. Est. & S2 (\%) & Desv. Est. \\
\hline Hombre & 95 & 40,4 & 93,74 & 3,85 & 91,39 & 5,16 \\
Mujer & 140 & 59,6 & 93,14 & 5,77 & 90,3 & 7,95 \\
Total & 235 & 100 & 93,38 & 5,08 & 90,75 & 6,96 \\
\hline
\end{tabular}


Tabla II. Promedio y desviación estándar de los índices S1 y S2 agrupados por rango etario.

\begin{tabular}{|c|c|c|c|c|c|c|}
\hline Años & $\mathrm{n}$ & $\%$ & S1 (\%) & Desv. Est. & S2 (\%) & Desv. Est. \\
\hline$>\mathrm{o}=\mathrm{a} 19$ & 52 & 22,1 & 92,76 & 5,11 & 89,94 & 6,99 \\
\hline $20-29$ & 150 & 63,8 & 93,51 & 4,92 & 90,91 & 6,78 \\
\hline $30-39$ & 22 & 9,4 & 93,47 & 6,53 & 90,89 & 8,82 \\
\hline $40-49$ & 8 & 3,4 & 95,57 & 3,79 & 93,58 & 5,40 \\
\hline $50-59$ & 3 & 1,3 & 91,43 & 3,80 & 88,65 & 5,19 \\
\hline Total & 235 & 100 & 93,38 & 5,08 & 90,75 & 6,96 \\
\hline
\end{tabular}

\section{DISCUSIÓN}

Los resultados obtenidos nos señalan que los promedios de la muestra estudiada sobre la proporción entre el cóndilo y la rama (S1) y la proporción entre cóndilo y mandíbula (S2), son similares a los resultados descritos por Kjellbert $e t$ $a l$. En la muestra de nuestro estudio se observó un predominio de radiografías pertenecientes a mujeres, además de un predominio de pacientes entre los 20 y 29 años, características de sexo y edad similares al estudio realizado por Miller $e t$ al. (1998) y en donde se encontraron pacientes sin trastornos témporomandibulares más simétricos que pacientes con este tipo de alteraciones. Los promedios encontrados para S1 y S2 en cuanto a sexo, muestran que tanto hombres como mujeres presentan simetrías en ambos índices, sin presentar diferencias significativas, de igual forma a lo sucedido en el estudio realizado por Ferrario et al. (1997), en donde los cóndilos medidos de hombres y mujeres también fueron simétricos.

En cuanto a la edad, los rangos descritos en este estudio, demuestran que tanto los grupos de 20 a 29,30 a 39 y 40 a 49 años presentan promedios de $\mathrm{S} 1$ y $\mathrm{S} 2$ compatibles con simetrías, sin encontrar diferencias significativas entre los grupos ( $\mathrm{p}=0,347$ y $\mathrm{p}=0,233$, para $\mathrm{S} 1$ y $\mathrm{S} 2$ respectivamente). Esto coincide con lo reportado por Alfaro et al., quienes describen que simetrías de cóndilo y rama no presentan diferencias significativas al ser comparadas con la edad de los pacientes, donde además los promedios de edades de los pacientes estudiados fueron similares a los presentes en este estudio.

Es importante considerar que a diferencia de estudios realizados previamente, el presente estudio considera RPN digitales sin diferenciar entre individuos sanos o individuos con alguna alteración, y aun así, los resultados son similares a lo indicado por algunos autores que han definido grupos de control y grupos de pacientes con diferentes alteraciones témporomandibulares y cráneo-maxilofaciales (AbadSantamaría et al.; Arenas et al.; Iturriaga et al.; Benzuur et al.). Tampoco se consideraron condiciones específicas como la pérdida de primeros molares, tal como lo realizó Halicioglu et al. (2013) en donde se compararon individuos en normoclusión y con pérdida del primer molar, sin encontrar diferencias significativas. A pesar de las diferencias mencionadas en las investigaciones sus conclusiones son similares: el sexo como factor de asimetría no presenta diferencias significativas (Ramirez-Yañez et al.; Saglam \& Sanli, 2004; Kiki et al., 2007; Kilic et al., 2008; Kurt et al., 2008; Kasimoglu et al.; Nolte et al., 2015). Tampoco se encontró diferencias significativas entre las edades adultas y presencia de simetría (Ferrario et al.; Kilic et al.; Iturriaga et al.). Sin embargo, existe evidencia sobre diferencias significativas entre la edad temprana y presencia o ausencia de simetrías asociadas a corrección de maloclusiones durante el desarrollo (Arenas et al.), lo que sugiere la realización de estudios prospectivos en pacientes en tratamiento de maloclusiones que determinen una relación entre tratamiento de alteraciones durante el crecimiento y simetría condilar.

Un aspecto importante a considerar, es el número de la muestra utilizado en nuestro estudio $(n=235)$, el cual fue superior al utilizado en otras investigaciones similares, las cuales sugieren el uso de muestras más numerosas para reafirmar la existencia de simetrías en los pacientes estudiados (Abad-Santamaría et al.; Benzuur et al.). Otro aspecto relevante, es el hecho de la realización de medidas mediante el programa computacional ImageJ, el cual permite la obtención de mediciones más exactas comparadas con otras técnicas (Liukkonen et al., 2005; Ramirez-Yañez et al.; Lemos et al., 2014). La digitalización de la imagen puede determinar de manera más precisa los contornos de las estructuras y permite la ampliación de la imagen y el cambio de contraste cuando sea necesario (Kasimoglu et al.). Esta modalidad supone ventajas frente a otras técnicas como por ejemplo el uso de papel de trazar (Saglam, 2003; Saglam \& Sanli; Kilic et al.; Kurt et al.).

En conclusión, los promedios de simetría condilar obtenidos en este estudio, al ser comparadas con los porcentajes postulados por la técnica de Kjellberg, demostraron simetría tanto en hombres como en mujeres y también en edades adultas, sin demostrar diferencias significativas en cuanto a sexo y edad. Por otro lado, el uso de programas computacionales 
es un método preciso para realizar mediciones en RPN digitales, por lo que se sugiere su uso en futuros estudios en donde se requiera la medición de estructuras.

FUENTES, R.; ARELLANO-VILLALÓN, M.; SOTO-FAÚNDEZ, N.; DIAS, F. J.; NAVARRO, P. \& ARIAS, A. Condylar and mandibular symmetry indexes through digital panoramic radiographs in a sample of chileans patients. Int. J. Morphol., 36(3):854-858, 2018.

SUMMARY: Mandibular symmetry may be modified by various conditions. A number of studies have attempted to define parameters of "normality" and several techniques have been described using digital orthopantomography (OPG). The aim of this study was to determine mandibular/condylar symmetry indexes in a sample of Chilean patients. A cross sectional descriptive study in digital OPG obtained from the Dental Clinic of Universidad de La Frontera was performed. Inclusion criteria applied were: Patients older than 18 years, complete permanent dentition up to the first molar. Exclusion criteria were also applied: Radiographic signs of bone disease and/or mandibular surgery, not visible condyles, orthodontics. ImageJ software was used to draw lines and measure the height of the ramus and mandibular condyle on the right and left side with the technique postulated by Kjellberg (1994). With these measurements, the symmetry index between condylar height and ramus ( 1 1) and between condylar and mandibular height (S2) were calculated. The study analyzed 235 digital OPG (95 men, 140 women) with an average age of $24.98 \pm 7.32$ years. The S1 average was $93.74 \%$ ( \pm 3.85$)$ for men and $93.41 \%( \pm 5.77)$ for women, and for S2 it was $90.50 \%( \pm 6.51)$ for men and $90.27 \%( \pm 7.80)$ for women, without significant differences for either index ( $\mathrm{p}=0.347$ and $\mathrm{p}=0.233$, respectively). No significant differences were found in reference to age, for indexes $\mathrm{S} 1$ and $\mathrm{S} 2$ ( $\mathrm{p}=0.594$ and $\mathrm{p}=0.669$, respectively). Therefore, it is concluded that there is presence of condylar/mandibular symmetry in both sexes and in age ranges, when measuring digital OPGs without considering health status or presence of any morphological or functional alteration.

KEY WORDS: Mandibular symmetry; Condylar symmetry; Digital panoramic radiographs; Prevalence.

\section{REFERENCIAS BIBLIOGRÁFICAS}

Abad-Santamaría, L.; López-de-Andrés, A.; Jiménez-Trujillo, I.; Ruíz, C. \& Romero, M. Effect of unilateral posterior crossbite and unilateral cleft lip and palate on vertical mandibular asymmetry. Ir. J. Med. Sci., 183(3):35762, 2014.

Alfaro, C.; Ayala, R.; Barrientos, S. S. \& Rodríguez, C. A. Mandibular asymmetries prevalence in panoramic radiographies in a Bogotá-Colombia population. Int. J. Morphol., 34(4):1203-6, 2016.

Arenas, C. S.; Araya-Díaz, P. \& Palomino, M. H. Evaluation of mandibular asymmetry in unilateral and bilateral posterior crossbite patients. Int. J. Morphol., 30(3):883-90, 2012.

Bezuur, J. N.; Habets, L. L. \& Hansson, T. L. The recognition of craniomandibular disorders--a comparison between clinical, tomographical, and dental panoramic radiographical findings in thirty-one subjects. J. Oral Rehabil., 15(6):549-54, 1988.

Ferrario, V. F.; Sforza, C.; Miani, A. Jr. \& Sigurtà, D. Asymmetry of normal mandibular condylar shape. Acta Anat. (Basel), 158(4):266-73, 1997.
Fuentes, R.; Engelke, W.; Bustos, L.; Oporto, G.; Borie, E.; Sandoval, P.; Garay, I.; Bizama, M. \& Borquez, P. Reliability of two techniques for measuring condylar asymmetry with x-rays. Int. J. Morphol., 29(3):694-701, 2011.

Habets, L. L.; Bezuur, J. N.; Naeji, M. \& Hansson, T. L. The Orthopantomogram, an aid in diagnosis of temporomandibular joint problems. II. The vertical symmetry. J. Oral Rehabil., 15(5):465-71, 1988.

Halicioglu, K.; Celikoglu, M.; Caglaroglu, M.; Buyuk, S. K.; Akkas, I. \& Sekerci, A. E. Effects of early bilateral mandibular first molar extraction on condylar and ramal vertical asymmetry. Clin. Oral Investig., 17(6):1557-61, 2013.

Iturriaga, V.; Navarro, P.; Cantín, M. \& Fuentes, R. Prevalence of vertical condilar asymmetry of the temporomandibular joint in patients with signs and symptoms of temporomandibular disorders. Int. J. Morphol., 30(1):31521, 2012.

Kasimoglu, Y.; Tuna, E. B.; Rahimi, B.; Marsan, G. \& Gencay, K. Condylar asymmetry in different occlusion types. Cranio, 33(1):10-4, 2015.

Kiki, A.; Kiliç, N. \& Oktay, H. Condylar asymmetry in bilateral posterior crossbite patients. Angle Orthod., 77(1):77-81, 2007.

Kilic, N.; Kiki, A. \& Oktay, H. Condylar asymmetry in unilateral posterior crossbite patients. Am. J. Orthod. Dentofacial Orthop., 133(3):382-7, 2008.

Kjellberg, H.; Ekestubbe, A.; Kiliaridis, S. \& Thilander, B. Condylar height on panoramic radiographs. A methodologic study with a clinical application. Acta Odontol. Scand., 52(1):43-50, 1994.

Kurt, G.; Uysal, T.; Sisman, Y. \& Ramoglu, S. I. Mandibular asymmetry in Class II subdivision malocclusion. Angle Orthod., 78(1):32-7, 2008.

Lemos, A. D.; Katz, C. R.; Heimer, M. V. \& Rosenblatt, A. Mandibular asymmetry: a proposal of radiographic analysis with public domain software. Dental Press J. Orthod., 19(3):52-8, 2014.

Liukkonen, M.; Sillanmäki, L. \& Peltomäki, T. Mandibular asymmetry in healthy children. Acta Odontol. Scand., 63(3):168-72, 2005.

Luz, J. G.; Miyazaki, L. T. \& Rodrigues, L. Verification of the symmetry of the mandibular ramus in patients with temporomandibular disorders and asymptomatic individuals: a comparative study. Bull. Group Int. Rech. Sci. Stomatol. Odontol., 44(3):83-7, 2002.

Miller, V. J.; Yoeli, Z.; Barnea, E. \& Zeltser, C. The effect of parafunction on condylar asymmetry in patients with temporomandibular disorders. J. Oral Rehabil., 25(9):721-4, 1998.

Nolte, J. W.; Karssemakers, L. H.; Grootendorst, D. C.; Tuinzing, D. B. \& Becking, A. G. Panoramic imaging is not suitable for quantitative evaluation, classification, and follow up in unilateral condylar hyperplasia. Br. J. Oral Maxillofac. Surg., 53(5):446-50, 2015.

Ramirez-Yañez, G. O.; Stewart, A.; Franken, E. \& Campos, K. Prevalence of mandibular asymmetries in growing patients. Eur. J. Orthod., 33(3):236$42,2010$.

Saglam, A. A. \& Sanli, G. Condylar asymmetry measurements in patients with temporomandibular disorders. J. Contemp. Dent. Pract., 5(3):59-65, 2004.

Saglam, A. M. The condylar asymmetry measurements in different skeletal patterns. J. Oral Rehabil., 30(7):738-42, 2003.

Direccion para correspondencia:

Dr. Ramón Fuentes Fernández

Centro de Investigación en Ciencias Odontológicas

Facultad de Odontología

Universidad de La Frontera

Av. Francisco Salazar 01145

Temuco

CHILE

Email: ramon.fuentes@ufrontera.cl

Recibido : 25-12-2017

Aceptado: 13-03-2018 